\title{
Strategic interaction and dynamics under endogenous time preference
}

\author{
Carmen Camacho ${ }^{\mathrm{a}, *}$, Cagri Saglam ${ }^{\mathrm{b}}$, Agah Turan ${ }^{\mathrm{b}}$ \\ ${ }^{a}$ CNRS and Université de Paris 1 Pantheon-Sorbonne, France \\ ${ }^{\mathrm{b}}$ Department of Economics, Bilkent University, Turkey
}

\section{A R T I C L E I N F O}

\section{Article history:}

Received 23 November 2011

Received in revised form

24 April 2013

Accepted 11 May 2013

Available online 27 May 2013

\section{Keywords:}

Supermodular games

Strategic growth

Lattice programming

Endogenous time preference

\begin{abstract}
A B S T R A C T
This paper presents a strategic growth model with endogenous time preference. Due to the potential lack of concavity and the differentiability of the value functions associated with each agent's problem, we employ the theory of monotone comparative statics and supermodular games based on order and monotonicity properties on lattices. In particular, we provide the sufficient conditions of supermodularity for dynamic games with open-loop strategies based on two fundamental elements: the ability to order elements in the strategy space of the agents and the strategic complementarity which implies upward sloping best responses. The supermodular game structure of the model lets us provide the existence and the monotonicity results on the greatest and the least equilibria. We sharpen these results by showing the differentiability of the value function and the uniqueness of the best response correspondences almost everywhere and show that the stationary state Nash equilibria tend to be symmetric. Finally, we numerically analyze to what extent the strategic complementarity inherent in agents' strategies can alter the convergence results that could have emerged under a single agent optimal growth model. In particular, we show that the initially rich can pull the poor out of the poverty trap even when sustaining a higher level of steady state capital stock for itself.
\end{abstract}

(c) 2013 Elsevier B.V. All rights reserved.

\section{Introduction}

To account for development patterns that differ considerably among economies in the long run (Quah, 1996; Barro, 1997; Barro and Sala-i-Martin, 1991), a variety of one-sector optimal growth models that incorporate some degree of market imperfections have been presented. These are based on technological external effects and increasing returns (Dechert and Nishimura, 1983; Mitra and Ray, 1984) or the endogeneity of time preference (Becker and Mulligan, 1997; Stern, 2006; Erol et al., 2011). They have characterized the optimal paths and proven the emergence of threshold dynamics according to which the economies with low initial capital stocks or incomes converge to a steady state with low per capita income, while economies with high initial capital stocks converge to a steady state with high per capita income (see Azariadis and Stachurski, 2005 for a survey). However, to what extent these analyses are robust to the considerations of strategic interactions among agents in the economy still remains a concern.

\footnotetext{
* Corresponding author. Tel.: +33 32486888553.

E-mail addresses: maria.camacho-perez@univ-paris1.fr (C. Camacho), csaglam@bilkent.edu.tr (C. Saglam), agah@bilkent.edu.tr (A. Turan).
}

This paper presents a strategic growth model with endogenous time preference. Each agent, receiving a share of income which is increasing in her own capital stock and decreasing in her rival's, invests over an infinite horizon to build her stocks. The heterogeneity among agents arises from differences in their initial endowment, their share of aggregate income, and therefore in their subjective discount rates. We adopt the non-cooperative openloop Nash equilibrium concept, in which players choose their strategies as simple time functions and they are able to commit themselves to time paths as equilibrium strategies. In this setup, agents choose their strategies simultaneously and each agent is faced with a single criterion optimization problem constrained by the strategies of the rival taken as given. We focus on the qualitative properties of the open-loop Nash equilibria and the dynamic implications of the strategic interaction.

In line with the empirical studies concluding that the rich are more patient than the poor (see Lawrence, 1991, Samwick, 1998) and in parallel to the idea that the stock of wealth is a key to reaching better health services and better insurance markets, we consider that the discount factor is increasing in the stock of wealth. However, this implies that the objective function of each agent's single criterion optimization problem includes a multiplication of the discount function. This generally destroys the usual concavity argument which is used in the proof of the differentiability of value 
function and the uniqueness of the optimal paths (see Benveniste and Scheinkman, 1979, Araujo, 1991).

Due to this potential lack of concavity and the differentiability of the value functions associated with each agent's problem, we employ the theory of monotone comparative statics and the supermodular games based on order and monotonicity properties on lattices (see Topkis, 1998). The analyses on the properties of supermodular games have been extensively concentrated in static games and to some extent in dynamic games with stationary Markov strategies (see Cooper, 1999, Amir, 2005, Vives, 2005 for a general review). This may stem from the fact that the use of open-loop strategies has been noted for being static in nature, not allowing for genuine strategic interaction between players during the playing of the game. There are, however, many situations in which players lack any other information than their own actions and time so that the open-loop strategies can turn out to be unavoidable. The players may be unable to observe the state vector, let alone the actions of their rivals. In this respect, showing how the supermodular game structure can be utilized in the analysis of the dynamic games under open-loop strategies is inevitable.

In this paper, we first provide the sufficient conditions of supermodularity for dynamic games with open-loop strategies based on two fundamental elements: the ability to order elements in the strategy space of the agents and the strategic complementarity which implies upward sloping best responses. In our dynamic game the open-loop strategies are vectors instead of simple scalars. Hence, the game requires an additional restriction to guarantee that all components of an agent's best response vector move together. This explains the role of the restriction that the payoff function of each agent has to be supermodular in his own strategy given the strategy of his rival. The supermodular game structure of our model lets us provide the existence and the monotonicity results on the greatest and the least equilibria. We sharpen these results by showing the differentiability of the value function and the uniqueness of the best response correspondences almost everywhere. These allow us to derive conclusions on the nature of best responses, the set of equilibria and the long-run dynamics.

In particular, we analyze to what extent the strategic complementarity inherent in agents' strategies can alter the convergence results that could have emerged under a single agent optimal growth model and try to answer the following questions: Can an agent with a larger initial stock credibly maintain this advantage to preempt the rival's investment and reach a better long-run stock of capital? Put differently, is the initial dominance reinforced by the actions of the agents? Can small initial differences be magnified and then propagated through time? Can this kind of initial advantages vanish in the non-cooperative equilibrium of this class of games with strategic complementarity? Can the agent with a low initial capital stock pull the rich to her lower steady state that she would never face while acting by herself? Under what conditions do we have a unique equilibrium with strategic complementarity under open-loop strategies?

The key feature of our analysis is that the stationary state Nash equilibria tend to be symmetric under open-loop strategies. We show that the initially rich can pull the poor out of the poverty trap even when sustaining a higher level of steady state capital stock for itself. A remarkable feature of our analysis is that it does not rely on particular parameterization of the exogenous functions involved in the model, rather, it provides a more flexible framework in regard to the discounting of time, keeps the model analytically tractable and uses only general and plausible qualitative properties.

The article is organized as follows. The next section introduces the model. Tools needed while utilizing the supermodularity of the game, equilibrium dynamics and the steady state analysis have been discussed in Section 3.

\section{Model}

We consider an intertemporal one sector model of a private ownership economy à la Arrow-Debreu with a single good $x_{t}$, and two infinitely lived agents, $i=1,2$. The single commodity is used as capital, along with labor, to produce output. Labor is presumed to be supplied in fixed amounts, and the capital and consumption are interpreted in per capita terms. The production function is given by $f\left(x_{t}\right)$. We assume that each agent receives a share of income $\theta^{i}\left(x_{t}^{i}, x_{t}^{j}\right)=\frac{x_{t}^{i}}{x_{t}^{i}+x_{t}^{j}}$, which is increasing in her own capital stock $x_{t}^{i}$, and decreasing in her rival's, $x_{t}^{j}$. The amount of current resources not consumed is saved individually as capital until the next period. The amount of current resources not consumed is saved individually as capital until the next period. For a given strategy of the rival, each agent chooses a path of consumption $c^{i}=$ $\left\{c_{t}^{i}\right\}_{t>0}$ so as to maximize the discounted sum of instantaneous utilities, $\sum_{t=0}^{\infty}\left(\prod_{s=1}^{t} \beta\left(x_{s}^{i}\right)\right) u^{i}\left(c_{t}^{i}\right)$ where the functions $u$ and $\beta$ denote the instantaneous utility from consumption and the level of discount on future utility, respectively.

In accordance with these, the problem of agent $i$ can be formalized as follows:

$$
\max _{\left\{c_{t}^{i}, x_{t+1}^{i}\right\}_{t=0}^{\infty}} \sum_{t=0}^{\infty}\left(\prod_{s=1}^{t} \beta\left(x_{s}^{i}\right)\right) u^{i}\left(c_{t}^{i}\right) \text {, }
$$

subject to

$c_{t}^{i}+x_{t+1}^{i} \leq \theta^{i}\left(x_{t}^{i}, x_{t}^{j}\right) f\left(x_{t}^{i}+x_{t}^{j}\right)+(1-\delta) x_{t}^{i}, \quad \forall t$,

$c_{t}^{i} \geq 0, \quad x_{t}^{i} \geq 0, \quad \forall t$,

$\left(x_{0}^{i}, x_{0}^{j}\right) \geq 0, \quad \boldsymbol{x}_{j}=\left\{x_{t}^{j}\right\}_{t=1}^{\infty} \geq 0, \quad$ given,

where $j \neq i \in\{1,2\}$, and $\delta \in(0,1)$ is the depreciation rate of the capital stock. Agents may only differ in their initial endowment, their share of output, and therefore in their subjective discount rates.

We make the following assumptions regarding the properties of the discount, utility and production functions.

Assumption 1. $\beta: \mathbb{R}_{+} \rightarrow \mathbb{R}_{++}$is continuous, differentiable, strictly increasing and satisfies $\sup _{x>0} \beta(x)=\beta_{m}<1, \sup _{x>0} \beta^{\prime}$ $(x)<+\infty$.

Assumption 2. $u: \mathbb{R}_{+} \rightarrow \mathbb{R}_{+}$is continuous, twice continuously differentiable and satisfies either $u(0)=0$ or $u(0)=-\infty$. Moreover, $u$ is strictly increasing, strictly concave and $u^{\prime}(0)=+\infty$ (Inada condition).

Assumption 3. $f: \mathbb{R}_{+} \rightarrow \mathbb{R}_{+}$is continuous, twice continuously differentiable and satisfies $f(0)=0$. Moreover, $f$ is strictly increasing and $\lim _{x \rightarrow+\infty} f^{\prime}(x)<\delta$.

We say that a path for capital $\boldsymbol{x}_{i}=\left(x_{1}^{i}, x_{2}^{i}, \ldots\right)$ is feasible from $\left(x_{0}^{i}, x_{0}^{j}\right) \geq 0$, if for all $t$ and given $\boldsymbol{x}_{j} \geq 0$, if for any $t \geq 0, \boldsymbol{x}_{i}$ satisfies that $0 \leq x_{t+1}^{i} \leq g\left(x_{t}^{i}, x_{t}^{j}\right)$ where

$g^{i}\left(x_{t}^{i}, x_{t}^{j}\right)=\theta^{i}\left(x_{t}^{i}, x_{t}^{j}\right) f\left(x_{t}^{i}+x_{t}^{j}\right)+(1-\delta) x_{t}^{i}$.

$S^{i}\left(\boldsymbol{x}_{j}\right)$ denotes the set of feasible accumulation paths from $\left(x_{0}^{i}, x_{0}^{j}\right)$. A consumption sequence $\boldsymbol{c}_{i}=\left(c_{0}^{i}, c_{1}^{i}, \ldots\right)$ is feasible from $\left(x_{0}^{i}\right.$, $\left.x_{0}^{j}\right) \geq 0$, when there exists a path for capital, $\boldsymbol{x}_{i} \in S^{i}\left(\boldsymbol{x}_{j}\right)$ with $0 \leq c_{t}^{i} \leq g^{i}\left(x_{t}^{i}, x_{t}^{j}\right)-x_{t+1}^{i}$. As the utility and the discount functions are strictly increasing, we introduce a function $U$ defined on the set of feasible sequences as

$U\left(\boldsymbol{x}_{i} \mid \boldsymbol{x}_{j}\right)=\sum_{t=0}^{\infty}\left(\prod_{s=1}^{t} \beta\left(x_{s}^{i}\right)\right) u\left(g^{i}\left(x_{t}^{i}, x_{t}^{j}\right)-x_{t+1}^{i}\right)$. 
The preliminary results are summarized in the following lemma which has a standard proof using the Tychonoff theorem (see Le Van and Dana, 2003; Stokey et al., 1989).

Lemma 1. Let $\bar{x}$ be the largest point $x \geq 0$ such that $f(x)+$ $(1-\delta) x=x$. Then, for any $\boldsymbol{x}_{i}$ in the set of feasible accumulation paths we have $x_{t}^{i} \leq A\left(x_{0}^{i}+x_{0}^{j}\right)$ for all $t$, where $A\left(x_{0}^{i}+x_{0}^{j}\right)=$ $\max \left\{\left(x_{0}^{i}+x_{0}^{j}\right), \bar{x}\right\}$. Moreover, the set of feasible accumulation paths is compact in the product topology defined on the space of sequences $\boldsymbol{x}_{i}$ and $U$ is well defined and upper semicontinuous over this set.

In a recent paper, Erol et al. (2011) study the dynamic implications of the endogenous rate of time preference depending on the stock of capital in a single consumer one-sector optimal growth model. They prove that even under a convex technology there exists a critical value of initial stock, in the vicinity of which, small differences lead to permanent differences in the optimal path: economies with low initial capital stocks converge to a steady state with low per capita income. On the other hand, economies with high initial capital stocks converge to a steady state with high per capita income. Indeed, it is shown that the critical stock is not an unstable steady state so that if an economy starts at this stock, an indeterminacy will emerge.

In this paper, we propose a capital accumulation game where heterogeneous agents consume strategically. Heterogeneity arises from differences in their initial endowment, their share of aggregate income, and therefore in their subjective discount rates. Our interest focuses on the qualitative properties of the openloop Nash equilibria and the dynamic implications of the strategic interaction.

\subsection{Non-cooperative difference game and open-loop Nash equilib- rium}

The non-cooperative game in consideration is a triplet $(N, \mathbf{S}$, $\left.\left\{U^{i}: i \in N\right\}\right)$ where $N=\{1,2\}$ is the set of players, $\mathbf{S}=\Pi_{i \in N} S^{i}$ is the set of joint admissible strategies under open-loop information structure and $U^{i}$ is the payoff function defined on $\mathbf{S}$ for each player $i \in N$, i.e., $U^{i}=U\left(\boldsymbol{x}_{i} \mid \boldsymbol{x}_{j}\right)$.

Any admissible strategy for agent $i$ is an infinite sequence compatible with the information structure of the game which is constant through time and restricted with the initial pair of capital stock in the economy. Accordingly, the set of admissible strategies for agent $i$ can be written as $S^{i}=\Pi_{t=1}^{\infty} S_{t}^{i}$, where $S_{t}^{i}=\left[0, g^{i}\left(\widetilde{x}_{t-1}^{i}, \widetilde{x}_{t-1}^{j}\right)\right]$, with $\widetilde{x}_{t-1}^{i}=\sup S_{t-1}^{i}$, and $\widetilde{x}_{t-1}^{j}=\sup S_{t-1}^{j}$. Indeed, any strategy $\boldsymbol{x}_{i} \in S^{i}$ is such that $x_{t}^{i} \in S_{t}^{i}, \forall t$ where $S_{1}^{i}=\left[0, g^{i}\left(x_{0}^{i}, x_{0}^{j}\right)\right], S_{2}^{i}=\left[0, g^{i}\left(g^{i}\left(x_{0}^{i}, x_{0}^{j}\right), g^{j}\left(x_{0}^{i}, x_{0}^{j}\right)\right)\right], \ldots$ etc.

A few important remarks on the way the set of joint admissible strategies is constructed are in order. Denoting the set of joint feasible strategies by $\Delta$, for any $\left(\boldsymbol{x}_{i}, \boldsymbol{x}_{j}\right) \in \Delta$, we have

$\Delta\left(\boldsymbol{x}_{i}, \boldsymbol{x}_{j}\right)=\left[\left(\cup_{\boldsymbol{x}_{j} \in X^{j}} S^{i}\left(\boldsymbol{x}_{j}\right)\right) \times\left(\cup_{\boldsymbol{x}_{i} \in X^{i}} S^{j}\left(\boldsymbol{x}_{i}\right)\right)\right] \cap \Delta$,

where $X^{j}=\left\{\boldsymbol{x}_{j}: S^{i}\left(\boldsymbol{x}_{j}\right) \neq \varnothing\right\}$. It is important to recall from Topkis (1998) that $\Delta=\left(\cup_{\boldsymbol{x}_{j} \in X^{j}} S^{i}\left(\boldsymbol{x}_{j}\right)\right) \times\left(\cup_{\boldsymbol{x}_{i} \in X^{i}} S^{j}\left(\boldsymbol{x}_{i}\right)\right)$ if and only if $\Delta\left(\boldsymbol{x}_{i}, \boldsymbol{x}_{j}\right)=\Delta$ for each $\left(\boldsymbol{x}_{i}, \boldsymbol{x}_{j}\right)$ in $\Delta$. However, note that under the open-loop information structure of our game, the action spaces of the agents turn out to be dependent on each other converting the game into a "generalized game" in the sense of Debreu (1952). More precisely, $\Delta \neq\left(\cup_{\boldsymbol{x}_{j} \in X^{j}} S^{i}\left(\boldsymbol{x}_{j}\right)\right) \times\left(\cup_{\boldsymbol{x}_{i} \in X^{i}} S^{j}\left(\boldsymbol{x}_{i}\right)\right)$ as the set of feasible accumulation paths from $\left(x_{0}^{i}, x_{0}^{j}\right)$ of agent $i$ is constrained by the choices of agent $j$. This simply prohibits us to order elements in the joint feasible strategy space and calls for additional restrictions on the plan of the game in proving the existence of an equilibrium and analyzing the long-run dynamics via order theoretical reasoning. The admissibility condition ${ }^{1}$ imposed on the set of feasible strategies of the agents allows us to write the set of joint admissible strategies as a simple cross product of each agent's set of admissible strategies that constitute a complete lattice. ${ }^{2}$

We adopt the non-cooperative open-loop Nash equilibrium concept, in which players choose their strategies as simple time functions and they are able to commit themselves to time paths as equilibrium strategies. In this setup, agents choose their strategies simultaneously and each agent is faced with a single criterion optimization problem constrained by the strategies of the rival taken as given.

For each vector $\boldsymbol{x}_{j} \in S^{j}$, the best response correspondence for agent $i$ is the set of all strategies that are optimal for agent $i$ given $\boldsymbol{x}_{j}$ :

$B r^{i}\left(\boldsymbol{x}_{j}\right)=\arg \max _{\boldsymbol{x}_{i} \in S^{i}} U\left(\boldsymbol{x}_{i} \mid \boldsymbol{x}_{j}\right)$

A feasible joint strategy $\left(\boldsymbol{x}_{i}^{*}, \boldsymbol{x}_{j}^{*}\right)$ is an open-loop Nash equilibrium if

$U\left(\boldsymbol{x}_{i}^{*} \mid \boldsymbol{x}_{j}^{*}\right) \geq U\left(\boldsymbol{x}_{i} \mid \boldsymbol{x}_{j}^{*}\right) \quad$ for each $\boldsymbol{x}_{i} \in S^{i}$ and each $i \in N$.

Given an equilibrium path, there is no feasible way for any agent to strictly improve its lifetime discounted utility as the strategies of the other agent remain unchanged. The set of all equilibrium paths for this non-cooperative game $\left(N, \mathbf{S},\left\{U^{i}: i \in N\right\}\right)$ is then identical to the set of pairs of sequences, $\left(\boldsymbol{x}_{i}^{*}, \boldsymbol{x}_{j}^{*}\right)$ such that

$\boldsymbol{x}_{i}^{*} \in B r^{i}\left(\boldsymbol{x}_{j}^{*}\right)$ and $\boldsymbol{x}_{j}^{*} \in B r^{j}\left(\boldsymbol{x}_{i}^{*}\right)$.

We will concentrate on the existence of such an equilibrium. To do so, we will first prove that the best response correspondence of each agent is nonempty so that there exists an optimal solution to problem $(\mathcal{P})$. The dynamic properties of the best response correspondence then follows from the standard analysis in optimal growth models (see Stokey et al., 1989, Le Van and Dana, 2003, Erol et al., 2011).

\subsection{Dynamic properties of the best response correspondence}

The existence of an optimal path associated with $(\mathcal{P})$ follows from the set of feasible accumulation paths being compact in the product topology defined on the space of sequences $\boldsymbol{x}_{i}$ and $U^{i}$ being upper semicontinuous for this product topology. Let $\boldsymbol{x}_{i} \in \mathrm{Br}^{i}\left(\boldsymbol{x}_{j}\right)$ so that $\boldsymbol{x}_{i}$ solves $(\mathcal{P})$ given $\boldsymbol{x}_{j}$. We can prove that the associated optimal consumption and capital paths are positive at equilibrium.

Proposition 1. Let $\boldsymbol{x}_{i} \in B r^{i}\left(\boldsymbol{x}_{j}^{*}\right)$.

(i) The associated optimal consumption path, $\boldsymbol{c}_{i}(i, j \in N, i \neq j)$, is given by

$$
c_{t}^{i}=g^{i}\left(x_{t}^{i}, x_{t}^{j *}\right)-x_{t+1}^{i}, \quad \forall t
$$

\footnotetext{
1 Whenever there exists a positive externality, i.e., $\frac{\partial g^{i}\left(x_{t}^{i}, x_{t}^{j}\right)}{\partial x_{t}^{j}} \geq 0, \forall t$, the admissibility imposed on the set of joint feasible strategies is far from a restriction. Even in case of negative externality, i.e., $\frac{\partial g^{i}\left(x_{t}^{i}, x_{t}^{j}\right)}{\partial x_{t}^{j}}<0, \forall t$, the long-run implications of our analysis will not rely on such a restriction. Indeed, an admissible strategy of agent $i$ is an infinite sequence of capital stock feasible from $\left(x_{0}^{i}, x_{0}^{j}\right)$ constituted under consideration of the highest feasible strategy of the rival.

2 In order to be able to work on a joint strategy space which constitutes a complete lattice, one may also introduce an ad hoc rule that exhausts the available stock of capital at the period where the joint strategies of the agents turn out to be infeasible (see Sundaram, 1989). However, in such a case, showing that the payoff function of each agent exhibits "increasing first differences" on the joint strategies turns out to be unnecessarily complicated.
} 
(ii) Given $\left\{x_{0}^{j}, \boldsymbol{x}_{j}^{*}\right\}$, if $x_{0}^{i}>0$, every solution $\left(\boldsymbol{x}_{i}, \boldsymbol{c}_{i}\right)$ to $(\mathcal{P})$ satisfies

$$
c_{t}^{i}>0, \quad x_{t}^{i}>0, \quad \forall t .
$$

Proof. It can be easily checked from the first order conditions and the Inada condition.

In accordance with these, let the value function $V$ associated with $(\mathcal{P})$ be defined by

$\forall x_{0}^{i} \geq 0, \quad V\left(x_{0}^{i} \mid\left\{x_{0}^{j}, \boldsymbol{x}_{j}^{*}\right\}\right)=\max _{\boldsymbol{x}_{i} \in S^{i}} U\left(\boldsymbol{x}_{i} \mid \boldsymbol{x}_{j}^{*}\right)$.

The bounds on discounting together with the existence of a maximum sustainable capital stock guarantee a finite value function. Under Assumptions 1 and 2, one can immediately show that the value function is non-negative and strictly increasing. If $u(0)=0$ then the continuity of the value function immediately follows as well. If $u(0)=-\infty$ then the value function turns out to be continuous in the generalized sense so that it is continuous at any strictly positive point and it converges to $-\infty$ when the stock of capital converges to zero (see Le Van and Dana, 2003). Given these, the Bellman equation associated with $(\mathcal{P})$ follows.

Proposition 2. (i) $V$ satisfies the following Bellman equation:

$$
\begin{aligned}
\forall x_{0}^{i} \geq & 0, \quad V\left(x_{0}^{i} \mid\left\{x_{0}^{j}, \boldsymbol{x}_{j}^{*}\right\}\right) \\
= & \max \left\{u\left(g^{i}\left(x_{0}^{i}, x_{0}^{j}\right)-x^{i}\right)\right. \\
& \left.+\beta\left(x^{i}\right) V\left(x^{i} \mid\left\{x_{0}^{j}, \boldsymbol{x}_{j}^{*}\right\}\right) \mid 0 \leq x^{i} \leq g^{i}\left(x_{0}^{i}, x_{0}^{j}\right)\right\} .
\end{aligned}
$$

(ii) A sequence $\boldsymbol{x}_{i} \in S^{i}\left(\boldsymbol{x}_{j}^{*}\right)$ is an optimal solution so that $\boldsymbol{x}_{i} \in \operatorname{Br}^{i}\left(\boldsymbol{x}_{j}^{*}\right)$ if and only if it satisfies:

$$
\begin{aligned}
\forall t, \quad V\left(x_{t}^{i} \mid\left\{x_{0}^{j}, \boldsymbol{x}_{j}^{*}\right\}\right)= & u\left(g^{i}\left(x_{t}^{i}, x_{t}^{j^{*}}\right)-x_{t+1}^{i}\right) \\
& +\beta\left(x_{t+1}^{i}\right) V\left(x_{t+1}^{i} \mid\left\{x_{0}^{j}, \boldsymbol{x}_{j}^{*}\right\}\right) .
\end{aligned}
$$

Proof. See Le Van and Dana (2003) or Erol et al. (2011).

The optimal policy correspondence associated with $(\mathcal{P}), \mu^{i}$ : $\mathbb{R}_{+} \rightarrow \mathbb{R}_{+}$is defined as follows:

$$
\begin{aligned}
& \mu^{i}\left(x_{0}^{i} \mid\left\{x_{0}^{j}, \boldsymbol{x}_{j}^{*}\right\}\right) \\
& =\arg \max \left\{u\left(g^{i}\left(x_{0}^{i}, x_{0}^{j}\right)-x^{i}\right)\right. \\
& \left.\quad+\beta\left(x^{i}\right) V\left(x^{i} \mid\left\{x_{0}^{j}, \boldsymbol{x}_{j}^{*}\right\}\right) \mid x^{i} \in\left[0, g^{i}\left(x_{0}^{i}, x_{0}^{j}\right)\right]\right\} .
\end{aligned}
$$

It is important to note that although the utility function is strictly concave, the solution to $(\mathcal{P})$ may not be unique as the multiplication of a discount function destroys the concave structure needed for uniqueness. We can prove the following properties for the optimal policy correspondence associated with $(\mathcal{P})$.

Proposition 3. (i) $\mu^{i}\left(0 \mid\left\{x_{0}^{j}, \boldsymbol{x}_{j}^{*}\right\}\right)=\{0\}$.

(ii) If $x_{0}^{i}>0$ and $x_{1}^{i} \in \mu^{i}\left(x_{0}^{i} \mid\left\{x_{0}^{j}, \boldsymbol{x}_{j}^{*}\right\}\right)$, then $0<x_{1}^{i}<g^{i}\left(x_{0}^{i}, x_{0}^{j}\right)$.

(iii) $\mu^{i}$ is upper semicontinuous.

(iv) $\boldsymbol{x}_{i} \in B r^{i}\left(\boldsymbol{x}_{j}^{*}\right)$ if and only if $x_{t+1}^{i} \in \mu^{i}\left(x_{t}^{i} \mid\left\{x_{0}^{j}, \boldsymbol{x}_{j}^{*}\right\}\right), \forall t$.

(v) The optimal correspondence $\mu^{i}$ is increasing so that if $x_{0}^{i}<$ $\tilde{x}_{0}^{i}, x_{1}^{i} \in \mu^{i}\left(x_{0}^{i} \mid\left\{x_{0}^{j}, \boldsymbol{x}_{j}^{*}\right\}\right)$ and $\tilde{x}_{1}^{i} \in \mu^{i}\left(\tilde{x}_{0}^{i} \mid\left\{x_{0}^{j}, \boldsymbol{x}_{j}^{*}\right\}\right)$ then $x_{1}^{i}<\tilde{x}_{1}^{i}$.
Proof. (ii) Follows easily from (3). (iii) See Le Van and Dana (2003). (iv) Follows from (6). (v) See Dechert and Nishimura (1983) or Amir et al. (1991).

The increasingness of $\mu^{i}$ is crucial for the convergence of optimal paths associated with $(\mathcal{P})$ and hence for the analysis of the long-run dynamics. Moreover, we have also proven that the optimal correspondence, $\mu$, is not only closed but also upper semicontinuous.

With the positivity of the optimal consumption and the stock of capital, the Euler equation associated with $(\mathcal{P})$ easily follows.

Proposition 4. When $x_{0}^{i}>0$, any solution $\boldsymbol{x}_{i} \in \operatorname{Br}^{i}\left(\boldsymbol{x}_{j}^{*}\right)$ satisfies the Euler equation associated with $(\mathcal{P})$ for all $t$ :

$$
\begin{aligned}
& u^{\prime}\left(g^{i}\left(x_{t}^{i}, x_{t}^{j^{*}}\right)-x_{t+1}^{i}\right) \\
& =\beta\left(x_{t+1}^{i}\right) u^{\prime}\left(g^{i}\left(x_{t+1}^{i}, x_{t+1}^{j^{*}}\right)-x_{t+2}^{i}\right) \frac{\partial g^{i}\left(x_{t+1}^{i}, x_{t+1}^{j^{*}}\right)}{\partial x_{t+1}^{i}} \\
& \quad+\beta^{\prime}\left(x_{t+1}^{i}\right) V\left(x_{t+1}^{i} \mid\left\{x_{0}^{j}, \boldsymbol{x}_{j}^{*}\right\}\right) .
\end{aligned}
$$

Recall that in a standard optimal growth model with geometric discounting and the usual concavity assumptions on preferences and technology, the optimal policy correspondence is single valued. Furthermore, the properties of the optimal path are easily found using the first order conditions together with the envelope theorem, differentiating the value function. However, in the $i$ 'th agent's problem $(\mathcal{P})$, although the utility function is strictly concave, the solution, namely $\mathrm{Br}^{i}\left(\boldsymbol{x}_{j}^{*}\right)$, may not be unique as the objective function includes the multiplication of a discount function. This generally destroys the usual concavity argument in the proof of the differentiability of value function and the uniqueness of the optimal paths (see Benveniste and Scheinkman, 1979, Araujo, 1991). To this end, we show that the value function associated with the $i$ 'th agent's problem $(\mathcal{P})$ is differentiable almost everywhere so that there exists a unique path from almost everywhere.

Proposition 5. (i) If $\boldsymbol{x}_{i} \in \mathrm{Br}^{i}\left(\boldsymbol{x}_{j}^{*}\right)$, then $V$ is differentiable at any $x_{t}^{i}, t \geq 1$. If $\boldsymbol{x}_{i} \in B r^{i}\left(\boldsymbol{x}_{j}^{*}\right)$, there exists a unique optimal path from $x_{t}^{i}$ for any $t \geq 1$.

(ii) $V$ is differentiable almost everywhere, i.e. the optimal path is unique for almost every $x_{0}>0$.

Proof. See Le Van and Dana (2003).

We prove in the next proposition that the optimal paths associated with $(\mathcal{P})$ are monotonic. As a monotone real valued sequence will either diverge to infinity or converge to some real number, the monotonicity of the optimal capital sequences $\boldsymbol{x}_{i} \in$ $\mathrm{Br}^{i}\left(\boldsymbol{x}_{j}^{*}\right)$ will be crucial in the analysis of the dynamic properties and the long-run behavior of the best response correspondences.

Proposition 6. For any initial condition $\left(x_{0}^{i}, x_{0}^{j}\right)$, the optimal path $\boldsymbol{x}_{i} \in \mathrm{Br}^{i}\left(\boldsymbol{x}_{j}^{*}\right)$ is monotonic.

Proof. Since $\mu^{i}$ is increasing, if $x_{0}^{i}>x_{1}^{i}$, we have $x_{1}^{i}>x_{2}^{i}$. Then, by induction, it is true that $x_{t}^{i}>x_{t+1}^{i}, \forall t$. If $x_{1}^{i}>x_{0}^{i}$, using the same argument yields $x_{t+1}^{i}>x_{t}^{i}, \forall t$. Now if $x_{1}^{i}=x_{0}^{i}$, then $x_{0}^{i} \in$ $\mu^{i}\left(x_{0}^{i} \mid\left\{x_{0}^{j}, \boldsymbol{x}_{j}^{*}\right\}\right)$. Recall that there exists a unique equilibrium path from $x_{t}^{i}$ for any $t \geq 1$. Since $x_{0}^{i} \in \mu^{i}\left(x_{0}^{i} \mid\left\{x_{0}^{j}, \boldsymbol{x}_{j}^{*}\right\}\right), x_{t}^{i}=$ $x_{0}^{i}, \forall t$. 
We will now present the condition under which the convergence to a steady state is guaranteed and concentrate on the behavior of the optimal paths $\boldsymbol{x}_{i} \in B^{i}\left(\boldsymbol{x}_{j}^{*}\right)$ associated with $(\mathcal{P})$.

Proposition 7. (i) There exists an $\xi>0$ such that if $\sup _{x>0} f^{\prime}(x)<$ $\frac{1-\xi}{\beta_{m}}$, then any optimal path $\boldsymbol{x}_{i} \in B r^{i}\left(\boldsymbol{x}_{j}^{*}\right)$ converges to zero.

(ii) Assume $x_{0}^{i}>0$. Let $\inf _{x>0} \beta(x)=\underline{\beta}$. If $\frac{\partial g^{i}\left(0, x_{0}^{j^{*}}\right)}{\partial x_{0}^{i}}>\frac{1}{\beta}$, then the optimal path $\boldsymbol{x}_{i}^{*} \in B^{i}\left(\boldsymbol{x}_{j}^{*}\right)$ converges to a steady state $x^{i}>0$.

Proof. See Erol et al. (2011).

We will now concentrate on the existence of an open-loop Nash equilibrium to the non-cooperative game $\left(N, \mathbf{S},\left\{U^{i}: i \in N\right\}\right)$. To achieve our goal, we will show that the non-cooperative game $\left(N, \mathbf{S},\left\{U^{i}: i \in N\right\}\right)$ is a supermodular game under openloop strategies. Besides, we will prove that under some regularity conditions, the set of equilibria is a nonempty complete lattice.

\section{Supermodular games and the existence of Nash equilibrium}

Let us first outline the fundamental properties of the supermodular games:

Definition 1 (Topkis, 1998). A non-cooperative game $\left(N, \mathbf{S},\left\{U^{i}\right.\right.$ : $i \in N\}$ ) is a supermodular game if the set $\mathbf{S}$ of admissible joint strategies is a sublattice of $\mathbb{R}^{m}$ (or of $\prod_{i \in N} \mathbb{R}^{m_{i}}$ ), and if for each $i, j \in N, i \neq j$, the payoff function $U^{i}$ is supermodular in $\boldsymbol{x}_{i}$ on $S^{i}$ for each $\boldsymbol{x}_{j}$ in $S^{j}$ and $U^{i}$ has increasing differences in $\left(\boldsymbol{x}_{i}, \boldsymbol{x}_{j}\right)$ on $S^{i} \times S^{j}$

These hypotheses on the payoff function for each agent $i$ imply that any two components of agent $i$ 's strategy are complements and each component of $i$ 's strategy is complementary with any component of $j$ 's strategy. The following theorem provides the existence of extremal equilibria in supermodular games with modest regularity conditions.

Theorem 1 (Topkis, 1998). Consider a supermodular non-cooperative game $\left(N, \mathbf{S},\left\{U^{i}: i \in N\right\}\right)$ for which the set $\mathbf{S}$ of admissible joint strategies is nonempty, compact and for each $i, j \in N, i \neq j$, the payoff function $U^{i}$ is upper semicontinuous in $\boldsymbol{x}_{i}$ on $S^{i}\left(\boldsymbol{x}_{j}\right)$ for each $\boldsymbol{x}_{j}$ in $S^{j}$, then the set of equilibria is a nonempty complete lattice and $a$ greatest and a least equilibrium exist.

There are two fundamental elements in supermodular games: the ability to order elements in the strategy space of the agents and the strategic complementarity, which implies upward sloping best responses. These properties of supermodular games have been extensively used in static games and to some extent in dynamic games with Markov perfect strategies (see Cooper, 1999, Amir, 2005, Vives, 2005 for a general review). We already stated in the introduction that open-loop strategies do not allow for genuine interaction between the players during the game. However, they are most appropriate in situations where the information about the other players is reduced to their initial condition or games with a very large number of players. We can add games in which agents decide whether to enter or not a coalition. Suppose a number of players who evaluate the benefits of entering a coalition for a given period of time. They would set the rules of the coalition at the time the decision is taken. To evaluate the coalition, players would play open-loop strategies. For all these reasons, we believe it is important to show how the supermodular game structure can be utilized in the analysis of dynamic games under open-loop strategies.

Here we consider a dynamic game with open-loop strategies and place related restrictions on strategy spaces and payoff functions which lead to ordered strategy sets and monotone best responses: as the other player selects higher strategies, the remaining player will as well. This will allow us to derive conclusions on the nature of best responses and the set of equilibria. To this end, the next proposition is crucial as it establishes the conditions under which our capital accumulation game turns out to be supermodular.

Proposition 8. The non-cooperative game $\left(N, \mathbf{S},\left\{U^{i}: i \in N\right\}\right)$ is a supermodular game if for each $i, j \in N, i \neq j$, and for all $t$,

$$
\begin{aligned}
& \frac{\partial^{2}\left[\beta\left(x_{t}^{i}\right) u\left(g^{i}\left(x_{t}^{i}, x_{t}^{j}\right)-x_{t+1}^{i}\right)\right]}{\partial x_{t}^{i} \partial x_{t+1}^{i}} \geq 0, \\
& \frac{\partial^{2}\left[\beta\left(x_{t}^{i}\right) u\left(g^{i}\left(x_{t}^{i}, x_{t}^{j}\right)-x_{t+1}^{i}\right)\right]}{\partial x_{t}^{i} \partial x_{t}^{j}} \geq 0 .
\end{aligned}
$$

The set of equilibria for this supermodular game is a nonempty complete lattice and there exist a greatest and a least equilibrium.

Proof. See the Appendix.

In this dynamic game with open-loop information structure strategies are vectors instead of simple scalars. Hence, the game requires an additional restriction to guarantee that all components of an agent's best response vector move together. This explains the role of the restriction (8) that the payoff function of each agent has to be supermodular in his own strategy given the strategy of his rival. Put differently, given the choice of the rival, the agent is better off combining high activity in one component of choice with high activity in another (see Cooper, 1999). The restriction (9) ensures that the gains to a higher strategy by one player increase with the strategy taken by the other so that the best responses turn out to be monotone. The key characteristic of a supermodular game, namely the presence of strategic complementarities is ensured by the restriction (9) which essentially implies the monotonicity property of the best responses.

Though the conditions (8) and (9) can be interpreted along general lines regarding the supermodularity of the non-cooperative dynamic games under open-loop information structure, a further refinement of the conditions will be useful in providing their limitations and economic interpretations to the full extent. Indeed, under Assumptions (1)-(3), the conditions (8) and (9) can be recast for each $i, j \in N, i \neq j$, and for all $t$ as

$$
\begin{aligned}
& \frac{\beta^{\prime}\left(x_{t}^{i}\right)}{\beta\left(x_{t}^{i}\right)}+\frac{u^{\prime \prime}\left(c_{t}^{i}\right)}{u^{\prime}\left(c_{t}^{i}\right)} \frac{\partial g^{i}\left(x_{t}^{i}, x_{t}^{j}\right)}{\partial x_{t}^{i}} \leq 0, \\
& \left(\frac{\beta^{\prime}\left(x_{t}^{i}\right)}{\beta\left(x_{t}^{i}\right)}+\frac{u^{\prime \prime}\left(c_{t}^{i}\right)}{u^{\prime}\left(c_{t}^{i}\right)} \frac{\partial g^{i}\left(x_{t}^{i}, x_{t}^{j}\right)}{\partial x_{t}^{i}}\right) \frac{\partial g^{i}\left(x_{t}^{i}, x_{t}^{j}\right)}{\partial x_{t}^{j}} \\
& \quad+\frac{\partial^{2} g^{i}\left(x_{t}^{i}, x_{t}^{j}\right)}{\partial x_{t}^{i} \partial x_{t}^{j}} \geq 0,
\end{aligned}
$$

respectively. Accordingly, the supermodularity of the non-cooperative game $\left(N, \mathbf{S},\left\{U^{i}: i \in N\right\}\right)$ crucially depends on the sensitivity of the agents' time preferences with respect to their stock of wealth and the sensitivity of the gains to a higher strategy by one player with respect to the rival's stock of capital. Note that for sufficiently low values of the marginal rate of patience, if the aggregate income of each player decreases with the rival's stock of capital, a sufficient condition for the supermodularity of the non-cooperative game turns out to be the supermodularity of the aggregate income of each agent, i.e. $\frac{\partial^{2} g^{i}\left(x_{t}^{i}, x_{t}^{j}\right)}{\partial x_{t}^{i} \partial x_{t}^{j}} \geq 0, \forall i, j \in N, i \neq j$, and $\forall t$. However, if the aggregate income of each player increases with the rival's stock of capital then the supermodularity of each agent's aggregate income becomes necessary for the game to be supermodular. 
Proposition 8 ensures the existence of an open-loop Nash equilibrium path in a supermodular game. The following proposition provides monotone comparative statics results.

Proposition 9. Let $T$ be a partially ordered set of parameters and $(\Gamma(\tau), \tau \in T)$ with $\Gamma(\tau)=\left(N, \mathbf{S}^{\tau},\left\{U^{\tau i}: i \in N\right\}\right)$ be a parameterized family of supermodular games. $S^{\tau}$ and $U^{\tau i}$ denote the dependence of $S$ and $U^{i}$ on parameter $\tau$. The set $S^{\tau}$ of admissible joint strategies is nonempty and compact for each $\tau$ in $T$ and is increasing in $\tau$ on $T$. If for each $i, j \in N, i \neq j$, and for all $t$,

$\frac{\partial^{2}\left[\beta\left(x_{t}^{i}, \tau\right) u\left(g^{i}\left(x_{t}^{i}, x_{t}^{j}, \tau\right)-x_{t+1}^{i}, \tau\right)\right]}{\partial x_{t}^{i} \partial \tau} \geq 0$,

then the greatest and the least equilibrium of game $\Gamma(\tau)$ are increasing in $\tau$ on $T$.

Proof. See the Appendix.

On a parameterized collection of supermodular games, the condition (10) ensures that the best response correspondence of the agents, hence the extremal equilibrium, will be increasing in a parameter that may be endogenous or exogenous due to the strategic complementarity inherent in the agents' strategies. As an example, consider that $\beta(x)=\eta-\gamma e^{-(x+\rho)^{\varepsilon}}$ where $0<$ $\gamma e^{-\rho^{\varepsilon}}<\eta<1,0<\varepsilon<1$, and $\rho>0$ (see Stern, 2006). Proposition 9 asserts that an increase in $\eta$, a measure of patience, leads to an increase in the extremal open-loop Nash equilibria of the supermodular game $\left(N, \mathbf{S}^{\eta},\left\{U^{\eta i}: i \in N\right\}\right)$. That is, the more patient agents are, the larger the individual's capital stock at every period in the greatest and the least equilibrium trajectories.

In what follows we will concentrate on the dynamic properties of the open-loop Nash equilibria of the supermodular game $\left(N, \mathbf{S},\left\{U^{i}: i \in N\right\}\right)$. Since the open-loop Nash equilibrium is weakly time consistent, instead of referring to the discrete time Hamiltonian, we will focus on the closed-loop representation of the equilibrium strategies and utilize (5), (6), and Proposition 7 in determining the dynamic properties of the equilibrium paths.

\subsection{Dynamic properties of open-loop Nash equilibrium and steady} state

Let $\left(\boldsymbol{x}_{i}^{*}, \boldsymbol{x}_{j}^{*}\right)$ be an open-loop Nash equilibrium from $\left(x_{0}^{i}, x_{0}^{j}\right)$ of the non-cooperative game $\left(N, \mathbf{S},\left\{U^{i}: i \in N\right\}\right)$. We denote by $\left\{x_{0}^{i}, \boldsymbol{x}_{i}^{*}\right\}$ the $i$ 'th agent's trajectory of capital stock at such an equilibrium path, i.e., $\left\{x_{0}^{i}, \boldsymbol{x}_{i}^{*}\right\}=\left(x_{0}^{i}, x_{1}^{i^{*}}, \ldots, x_{t}^{i^{*}}, \ldots\right)$. It is then clear that $\left(\boldsymbol{x}_{i}^{*}, \boldsymbol{x}_{j}^{*}\right)$ satisfies $(2)$. Hence $\boldsymbol{x}_{i}^{*}$ solves $(\mathcal{P})$ given $\boldsymbol{x}_{j}^{*}$ so that $x_{t+1}^{i} \in \mu^{i}\left(x_{t}^{i} \mid\left\{x_{0}^{j}, \boldsymbol{x}_{j}^{*}\right\}\right), \forall t$.

Let $\inf _{x>0} \beta(x)=\underline{\beta}$ and $\frac{\partial g^{i}\left(0, x_{0}^{j^{*}}\right)}{\partial x_{0}^{i}}>\frac{1}{\beta}$, for each $i, j \in N, i \neq j$. The optimal paths $\boldsymbol{x}_{i}^{*} \in B r^{i}\left(\boldsymbol{x}_{j}^{*}\right)$ and $\boldsymbol{x}_{j}^{*} \in B r^{j}\left(\boldsymbol{x}_{i}^{*}\right)$ converge to the steady state values $x_{i}>0$ and $x_{j}>0$, respectively. These steady state values solve the following stationary state Euler equations:

$$
\begin{aligned}
u^{\prime}\left(g^{i}\left(x^{i}, x^{j}\right)-x^{i}\right)= & \beta^{\prime}\left(x^{i}\right) \frac{u\left(g^{i}\left(x^{i}, x^{j}\right)-x^{i}\right)}{1-\beta\left(x^{i}\right)} \\
& +\beta\left(x^{i}\right) u^{\prime}\left(g^{i}\left(x^{i}, x^{j}\right)-x^{i}\right) \\
& \times \frac{\partial g^{i}\left(x^{i}, x^{j}\right)}{\partial x^{i}}, \quad \forall i, j \in N, i \neq j .
\end{aligned}
$$

However, it is important to note that the stationary sequences associated with each solution of (11) may not induce a steady state open-loop Nash equilibrium (see Dockner and Nishimura, 2001) unless they constitute a best reply to each other. A steady state open-loop Nash equilibrium $\left(\boldsymbol{x}_{i}, \boldsymbol{x}_{j}\right)$ is defined as the stationary sequences associated with a solution $\left(x_{i}, x_{j}\right)$ to (11) such that $\left\{x_{0}^{i}, \boldsymbol{x}_{i}\right\}=\left(x^{i}, x^{i}, \ldots, x^{i}, \ldots\right) \in \operatorname{Br}^{i}\left(\boldsymbol{x}_{j}\right)$ and $\left\{x_{0}^{j}, \boldsymbol{x}_{j}\right\}=\left(x^{j}, x^{j}, \ldots\right.$, $\left.x^{j}, \ldots\right) \in B r^{j}\left(\boldsymbol{x}_{i}\right)$.

If the game was symmetric, we could have already concluded that the two agents will end up with the same amount of physical capital in the long run. Indeed, if a supermodular game is symmetric, then a greatest and a least equilibrium exist and they are symmetric. Amir et al. (2008) show that monotonicity induces the greatest and the least equilibrium converge to the highest and the lowest symmetric steady states, respectively. A game is symmetric supermodular if on top of the usual conditions for supermodularity, the agents' strategy spaces are identical and

$U\left(\boldsymbol{x}_{i} \mid \boldsymbol{x}_{j}\right)=U\left(\boldsymbol{x}_{j} \mid \boldsymbol{x}_{i}\right), \quad \forall\left(\boldsymbol{x}_{i}, \boldsymbol{x}_{j}\right) \in \mathbf{S}$.

Neither of these latter conditions are met in our game. Strategy spaces coincide only when the initial conditions are identical for both of the agents. Furthermore, the second condition also fails at the first period since we have $u\left(g^{i}\left(x_{0}^{i}, x_{0}^{j}\right)-x_{1}^{i}\right) \neq$ $u\left(g^{j}\left(x_{0}^{j}, x_{0}^{i}\right)-x_{1}^{i}\right)$ for each $i, j \in N, i \neq j$. Despite the lack of symmetry in the game, we prove that our game always leads to symmetric steady states so that the initial wealth differences vanish in the long run.

Proposition 10. All steady state open-loop Nash equilibria of the supermodular game $\left(N, \mathbf{S},\left\{U^{i}: i \in N\right\}\right)$ are symmetric.

Proof. See the Appendix.

In the following proposition, we show that the individual level of capital stock at the lowest and the highest steady state open-loop Nash equilibrium is greater than the lowest and the highest steady states of the associated single agent optimal growth problem, respectively.

Proposition 11. Let $x_{L}$ and $x_{H}$ denote the lowest and the highest steady states of the optimal growth problem and $x_{L}^{o}$ and $x_{H}^{o}$ denote the lowest and the highest steady state open-loop Nash equilibrium of the non-cooperative game $\left(N, \mathbf{S},\left\{U^{i}: i \in N\right\}\right)$. If the game is supermodular then we have $x_{L} \leq x_{L}^{o}$ and $x_{H} \leq x_{H}^{o}$.

Proof. See the Appendix.

Next we exploit the supermodular structure of our game in identifying the most preferred steady state depending on the game elements.

Corollary 1. Let $(\hat{\boldsymbol{x}}, \hat{\boldsymbol{x}})$ and $(\check{\boldsymbol{x}}, \check{\boldsymbol{x}})$ denote the highest and the lowest symmetric stationary open-loop Nash equilibrium of the game $\left(N, \mathbf{S},\left\{U^{i}: i \in N\right\}\right)$. If

$\frac{\partial}{\partial x}\left(\frac{u(g(x, x)-x)}{1-\beta(x)}\right)>0$,

then $(\hat{\boldsymbol{x}}, \hat{\boldsymbol{x}})$ is the most preferred steady state open-loop Nash equilibrium. Otherwise, $(\check{\boldsymbol{x}}, \check{\boldsymbol{x}})$ will be the most preferred.

Proof. See the Appendix.

Recall that the stationary sequences associated with each solution of (11) may not constitute a steady state open-loop Nash equilibrium. This moves the concern on the number of solutions to the stationary Euler equations and among those that will be induced by a steady state open-loop Nash equilibrium. Since one cannot provide an analytical answer to this question, we will move on to the numerical analysis of our problem. The next section is devoted to this end. For given functional forms, we analyze the 
solutions to (11). In order to determine which of these indeed constitute a steady state open-loop Nash equilibrium, we employ the iterations of the Bellman operator (5) for the given stationary strategy of the rival. We compute the dynamics of the equilibrium paths from an initial condition, using the supermodular structure of the game on top of the iterations of the Bellman operator.

\section{Characterization of the long-run equilibria: numerical anal- ysis}

The analysis of the solutions to the stationary Euler equations and the determination of solutions induced by a stationary openloop Nash equilibrium cannot be carried out without specifying the forms of the utility, discount and production functions. In what follows, our analysis will be based on the functional forms specified in accordance with Stern (2006). The utility, production and discount functions are specified as

$u(c)=\frac{c^{1-\sigma}}{1-\sigma}$,

$f(x)=A x^{\alpha}+(1-\delta) x$,

$\beta(x)=\eta-\gamma e^{-(x+\rho)^{\varepsilon}}$,

where $0<\{A, \rho\}, 0<\alpha, \sigma, \varepsilon<1$, and $0<\gamma e^{-\rho^{\varepsilon}}<\eta<$ 1. Under these functional forms, in reference to Proposition 8 , a sufficient condition for our strategic growth model to become a supermodular game is:

$\frac{\beta^{\prime}\left(x_{t}^{i}\right)}{\beta\left(x_{t}^{i}\right)} \leq \frac{(\sigma-1)\left(x_{t}^{j}+\alpha x_{t}^{i}\right)+x_{t}^{i}}{x_{t}^{i}\left(x_{t}^{i}+x_{t}^{j}\right)}, \quad \forall t \geq 1$.

Condition (12) is trivially checked for $\sigma \geq 1$ when $\rho$ is sufficiently large, since $\frac{\beta^{\prime}}{\beta}$ is a decreasing function and its supremum equals $\frac{\gamma \varepsilon \rho^{-1+\varepsilon}}{\eta-\gamma e^{-\rho^{\varepsilon}}}$. In accordance with these, we utilize the following set of fairly standard coefficients as our benchmark parameterization:

$A=0.75, \quad \alpha=0.4, \quad \delta=0.03, \quad \sigma=1.5$,

$\eta=0.95, \quad \gamma=2.5, \quad \rho=4.5, \quad \varepsilon=0.99$,

under which the maximum sustainable level of capital stock turns out to be $A\left(x_{0}^{i}+x_{0}^{j}\right)=\max \left\{\left(x_{0}^{i}+x_{0}^{j}\right), \bar{x}\right\}$, where $\bar{x}$ is $213.747 .^{3}$

We prove in the first subsection of the following that strategic interaction removes indeterminacy, in case indeterminacy existed in the single agent optimal growth problem. Then, we continue our numerical analysis with the case in which multiplicity of equilibria persists in the long run.

\subsection{Strategic interaction removes indeterminacy}

Under our benchmark parameterization, we can prove numerically that strategic growth removes indeterminacy and implies global convergence towards a unique symmetric steady state. In order to provide a better exposition of this point, and to provide a basis of comparison for our strategic growth model, we first recall the analysis of Erol et al. (2011) and we concentrate on the dynamic implications of endogenous discounting in the single agent framework.

\footnotetext{
3 Our analysis utilizes a felicity function constrained to a negative domain. Schumacher (2011) shows that if the discount rate is endogeneized via a state variable, the domain of the felicity function should be constrained to a positive domain. In a negative domain, a higher stock of capital would have a negative impact on overall welfare. However, under our parameterization, even with a negative felicity function constrained to a negative domain, a higher stock of capital will have a positive impact on welfare. Indeed, our discount factor attributed to the utility of consumption at period $t$ increases with the level of capital stock, as in Becker and Mulligan (1997) and Stern (2006).
}

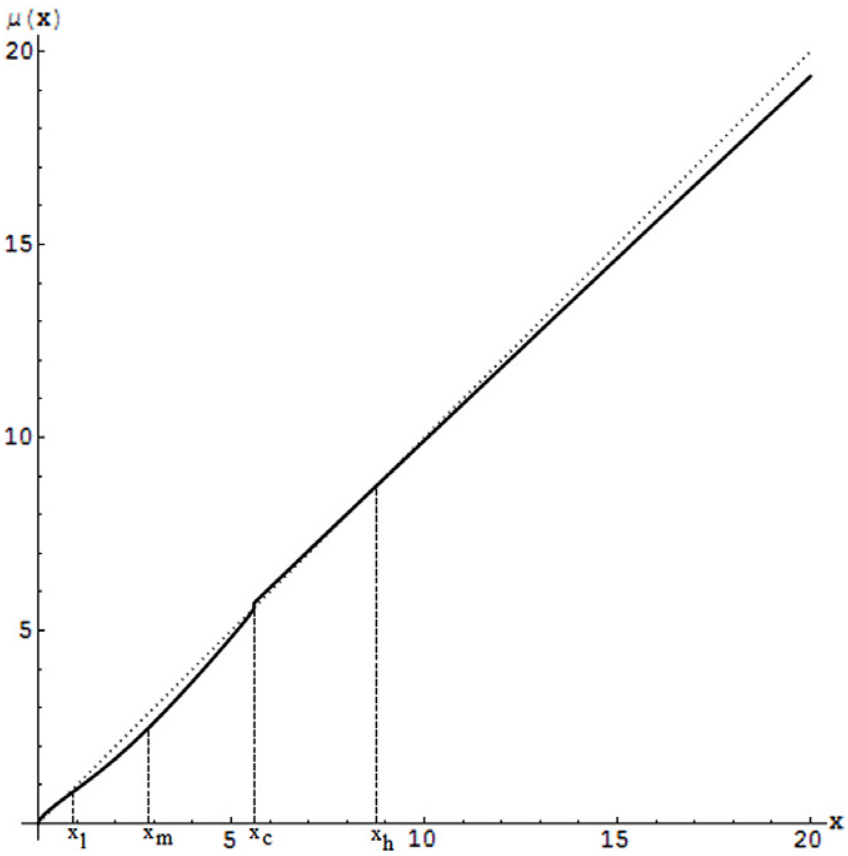

Fig. 1. Optimal policy after 300 iterations on the initial zero value function.

Case 1. Optimal growth framework.

Consider problem $(\mathcal{P})$ in which only agent $i$ starts with a positive stock, $x_{0}^{j}=0$ and $x_{0}^{i}>0$, i.e. agent $i$ acts alone. There exist three solutions to (11): $x_{l}=0.5953, x_{m}=2.9155$, and $x_{h}=8.4913$. In order to determine which of these are actually the optimal steady states, we analyze the optimal policy using the Bellman operator. Fig. 1 indicates that $x_{l}$ and $x_{h}$ are stable optimal steady states, but in contrast with Stern (2006), Fig. 1 strongly indicates that $x_{m}$ is not an optimal steady state. Indeed, if it were, the optimal policy would have crossed the $y=x$ line at $x_{m}$. The Bellman operator also reveals the existence of a genuine critical point at $x_{c} \approx 5.5846$ : for any initial capital stock level lower than $x_{c}$, the economy will face a development trap, enforcing convergence to a very low capital level $x_{l}$. On the other hand, for any initial capital level higher than $x_{c}$, the optimal path will converge to $x_{h}$. However, if an economy starts at $x_{c}$, an indeterminacy will emerge.

Case 2. Strategic growth.

Consider now the case where $\left(x_{0}^{i}, x_{0}^{j}\right)>0$. Let $\inf _{x>0} \beta(x)=$ $\underline{\beta}$. Note that $\frac{\partial g^{i}\left(0, x_{0}^{j^{*}}\right)}{\partial x_{0}^{i}}>\frac{1}{\beta}$. Under the benchmark parameter values, there exists a unique symmetric solution of the stationary state Euler equations $(11):\left(x_{i}^{*}, x_{j}^{*}\right)=(10.8906,10.8906)$. Indeed, consider a two-region economy, one with a large initial stock of capital and the other with almost none, take for example $x_{0}^{i}=0.1$ and $x_{0}^{j}=10$. Whenever they act independently of each other, the poor region ends up in a development trap, $x_{l}=0.5953$, whereas the rich region reaches a steady state level of capital stock $x_{h}=$ 8.4913. However, if there exists a strategic interaction between the two regions, they both reach an identical steady state level of capital $\left(x_{i}^{*}, x_{j}^{*}\right)=(10.8906,10.8906)$. Thanks to the strategic interaction between regions, the rich region pulls the poor one out of the poverty trap while sustaining a higher level of steady state capital stock in the rich region.

\subsection{Multiplicity of equilibria}

As we have already underlined, in a single agent optimal growth framework, the capital dependent time preference rate generates a 


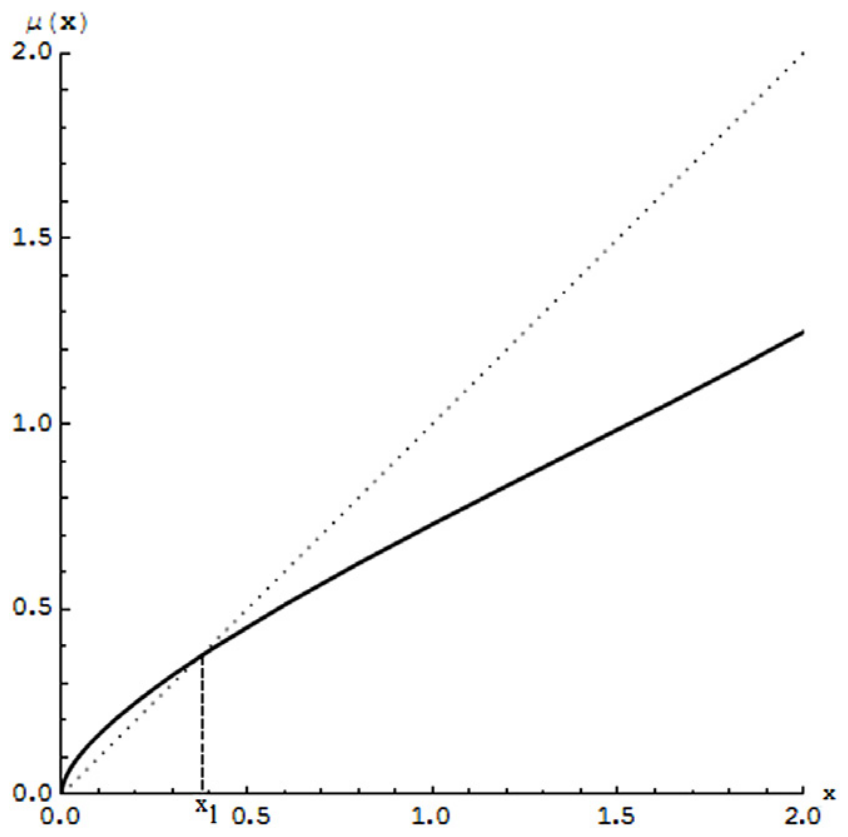

Fig. 2. Low steady state $\left(x_{l}=0.59\right)$ is optimal.

critical point. In the vicinity of this critical point, small differences lead to permanent differences in the optimal path. Since this result heavily depends on the value of $\rho$, we would like to deviate from the benchmark to explore how strategic interaction modifies the single agent result. Hence, we assign a lower value to $\rho, \rho=$ 4. The supermodular game $\left(N, \mathbf{S},\left\{U^{i}: i \in N\right\}\right)$ studied in this section exhibits multiple long-run equilibria although a single agent optimal growth model exhibits global convergence. Indeed, there exist three solutions to the stationary Euler equation (11) of the single agent optimal growth problem: $x_{l}=0.3708, x_{m}=$ 4.0061, and $x_{h}=8.4315$. Among these three solutions, $x_{l}$ turns out to be the only optimal steady state (see Fig. 2). The natural question is then to what extent strategic growth dynamics are affected from such a change? When we consider the dynamic implications of strategic growth we note that there are multiple solutions of the stationary state Euler equations as listed below. The stationary sequences associated with only those in bold are indeed constituting a steady state open-loop Nash equilibrium as we show later:

$\left(x_{i}, x_{j}\right)=\{(\mathbf{0 . 8 6 4}, \mathbf{0 . 8 6 4}),(2.2404,2.2404)$,

(10.8863, 10.8863), (2.7941, 1.1354),

(2.3869, 10.4927), (1.1353, 2.7941),

$(10.4927,2.3869)\}$.

The stationary sequences associated with the asymmetrical solutions above do not constitute a steady state open-loop equilibrium of our game as announced in Proposition 10. Among the three symmetric solutions to the stationary Euler equations (11), the stationary sequences associated with the lowest and the highest pairs constitute a steady state open-loop equilibrium, shown in Figs. 3 and 4 respectively. In other words, when the initial condition is $\left(x_{0}^{i}, x_{0}^{j}\right)=(10.8863,10.8863)$, then the stationary strategies of the agents sticking to the initial condition constitute a best reply to each other so that $(10.8863,10.8863)$ turns out to be a steady state open-loop equilibrium. The same is true for $(0.864,0.864)$. As we show in Proposition 11, both of them are higher than the unique steady state of the single agent optimal growth problem.

We can easily show that threshold dynamics emerge. There exist critical values of initial capital $\left(x_{c}, x_{c}\right)$, below which an

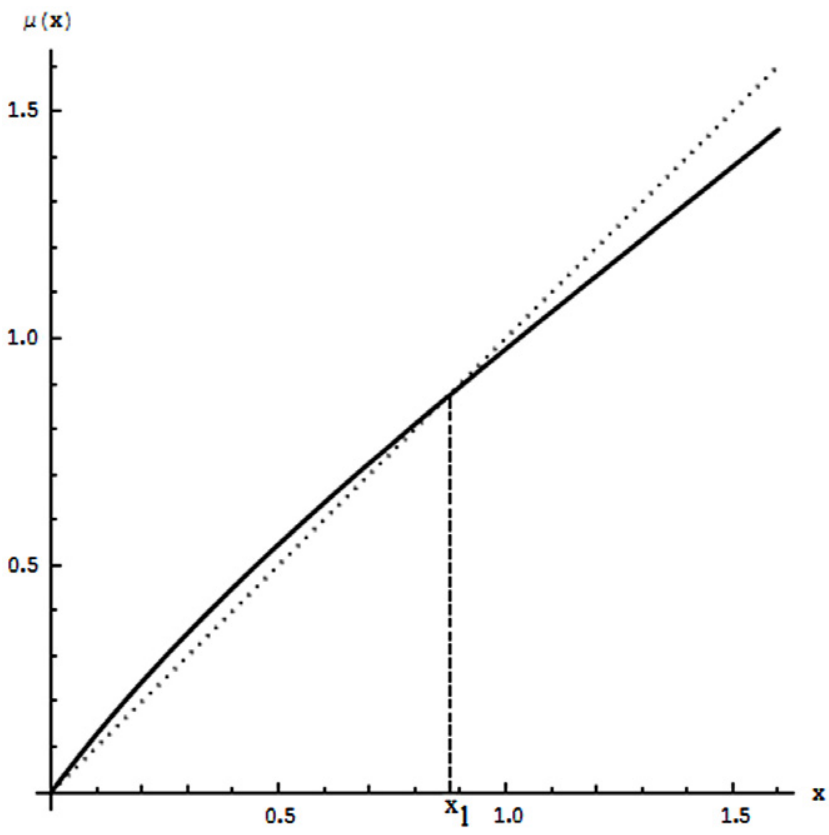

Fig. 3. Stationary sequence associated with $\left(x_{i}=0.863991\right)$ is an open-loop Nash equilibrium.

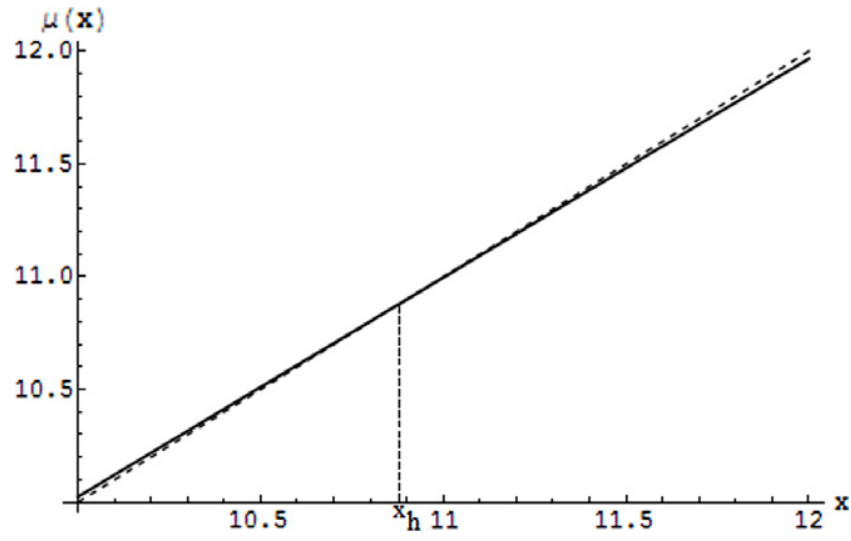

Fig. 4. Stationary sequence associated with $\left(x_{i}=10.8863\right)$ is an open-loop Nash equilibrium.

open-loop Nash equilibrium of our supermodular game will converge to the lowest steady state $(0.864,0.864)$. There exists a second critical point, $\left(x^{c}, x^{c}\right)$ above which a sequence of an open-loop Nash equilibrium will converge to the highest steady state $(10.8863,10.8863)$. Noteworthy, these critical values are not a solution of the stationary state Euler equations so that the stationary sequences associated with these cannot constitute a stationary state open-loop equilibrium of the game. As the optimal policy of agent $i$ is upper semicontinuous, given the strategy of the rival $\boldsymbol{x}_{j}^{*} \rightarrow 10.8863$, the graph of $\mu^{i}\left(x^{i} \mid\left\{x_{0}^{j}, \boldsymbol{x}_{j}^{*}\right\}\right)$ jumps over the $45^{\circ}$ line at $x^{c}$ so that $x^{c} \notin \mu^{i}\left(x^{c} \mid\left\{x_{0}^{j}, \boldsymbol{x}_{j}^{*}\right\}\right)$. With an analogous reasoning, given the strategy of the rival is $\boldsymbol{x}_{j}^{*} \rightarrow 0.864$, the graph of $\mu^{i}\left(x^{i} \mid\left\{x_{0}^{j}, \boldsymbol{x}_{j}^{*}\right\}\right)$ jumps over the $45^{\circ}$ line at $x_{c}$ so that $x_{c} \notin \mu^{i}\left(x_{c} \mid\left\{x_{0}^{j}, \boldsymbol{x}_{j}^{*}\right\}\right)$. This implies even further that as soon as $x^{c}=x_{c}$ an indeterminacy arises so that for a game emanating from such a critical stock of capital, the best responses of the two agents that converge either to the low or to the high steady state may both constitute an open-loop Nash equilibrium. 


\section{Appendix}

\section{A.1. Proof of Proposition 8}

The non-cooperative game $\left(N=\{1,2\}, \mathbf{S},\left\{U^{i}: i \in\{1,2\}\right\}\right)$ is supermodular if $\mathbf{S}$ is a sublattice of $\left(\prod_{i \in N} \mathbb{R}^{m_{i}}\right), U^{i}$ is supermodular in $\boldsymbol{x}_{i}$, for any $\boldsymbol{x}_{j}$ and $U^{i}$ has increasing differences in $\left(\boldsymbol{x}_{i}, \boldsymbol{x}_{j}\right)$.

(i) The lattice $\mathbf{S}$ is a subset of $\mathbb{R}^{\infty}$ and it is a sublattice of it.

(ii) Let us start proving that each individual payoff function is supermodular in its own strategy. By definition, a function $f$ is supermodular if and only if

$f(x \vee y)+f(x \wedge y) \geq f(x)+f(y)$.

Consider two different strategies for agent $i, \boldsymbol{x}_{i}$ and $\boldsymbol{x}_{i}^{\prime}$ who differ from each other at time $t$ and $t+1$ so that ${ }_{t+1} \boldsymbol{x}={ }_{t+1} \boldsymbol{x}_{i}^{\prime}=$ $\left(x_{t+2}^{i}, x_{t+3}^{i}, \ldots\right)$. Recall that

$U\left(t+1 \boldsymbol{x}_{\boldsymbol{i}} \mid \boldsymbol{x}_{j}\right)=\sum_{\tau=t+1}^{\infty}\left(\prod_{s=t+2}^{\tau} \beta\left(x_{s}^{i}\right)\right) u\left(g^{i}\left(x_{\tau}^{i}, x_{\tau}^{j}\right)-x_{\tau+1}^{i}\right)$.

We need to show that:

$$
\begin{aligned}
\beta\left(x_{t}^{\prime i}\right) u\left(g^{i}\left(x_{t}^{\prime i}, x_{t}^{j}\right)-x_{t+1}^{\prime i}\right)+\beta\left(x_{t}^{\prime i}\right) \beta\left(x_{t+1}^{\prime i}\right) U\left({ }_{t+1} \boldsymbol{x}_{\boldsymbol{i}}^{\prime} \mid \boldsymbol{x}_{j}\right) \\
+\beta\left(x_{t}^{i}\right) u\left(g^{i}\left(x_{t}^{i}, x_{t}^{j}\right)-x_{t+1}^{i}\right)+\beta\left(x_{t}^{i}\right) \beta\left(x_{t+1}^{i}\right) U\left({ }_{t+1} \boldsymbol{x}_{i} \mid \boldsymbol{x}_{j}\right) \\
\leq \beta\left(x_{t}^{\prime}\right) u\left(g^{i}\left(x_{t}^{\prime}, x_{t}^{j}\right)-x_{t+1}^{i}\right)+\beta\left(x_{t}^{\prime i}\right) \beta\left(x_{t+1}^{i}\right) U\left({ }_{t+1} \boldsymbol{x}_{i} \mid \boldsymbol{x}_{j}\right) \\
+\beta\left(x_{t}^{i}\right) u\left(g^{i}\left(x_{t}^{i}, x_{t}^{j}\right)-x_{t+1}^{\prime i}\right)+\beta\left(x_{t}^{i}\right) \beta\left(x_{t+1}^{i}\right) U\left({ }_{t+1} \boldsymbol{x}_{\boldsymbol{i}}^{\prime} \mid \boldsymbol{x}_{j}\right) .
\end{aligned}
$$

Since

$$
\begin{aligned}
& \left(\beta\left(x_{t}^{i}\right)-\beta\left(x_{t}^{\prime i}\right)\right) \beta\left(x_{t+1}^{\prime i}\right) U\left(_{t+1} \boldsymbol{x}_{\boldsymbol{i}}^{\prime} \mid \boldsymbol{x}_{j}\right) \\
& \quad \geq\left(\beta\left(x_{t}^{i}\right)-\beta\left(x_{t}^{\prime i}\right)\right) \beta\left(x_{t+1}^{i}\right) U\left({ }_{t+1} \boldsymbol{x}_{\boldsymbol{i}} \mid \boldsymbol{x}_{j}\right),
\end{aligned}
$$

it is sufficient to show that

$$
\begin{aligned}
& \beta\left(x_{t}^{\prime i}\right) u\left(g^{i}\left(x_{t}^{\prime i}, x_{t}^{j}\right)-x_{t+1}^{\prime i}\right)+\beta\left(x_{t}^{i}\right) u\left(g^{i}\left(x_{t}^{i}, x_{t}^{j}\right)-x_{t+1}^{i}\right) \\
& \quad \leq \beta\left(x_{t}^{\prime i}\right) u\left(g^{i}\left(x_{t}^{\prime i}, x_{t}^{j}\right)-x_{t+1}^{i}\right)+\beta\left(x_{t}^{i}\right) u\left(g^{i}\left(x_{t}^{i}, x_{t}^{j}\right)-x_{t+1}^{i}\right) .
\end{aligned}
$$

This is equivalent to showing that $\beta\left(x_{t}^{i}\right) u\left(g^{i}\left(x_{t}^{i}, x_{t}^{j}\right)-x_{t+1}^{i}\right)$ is supermodular in $\left(x_{t}^{i}, x_{t+1}^{i}\right)$. As $\beta\left(x_{t}^{i}\right) u\left(g^{i}\left(x_{t}^{i}, x_{t}^{j}\right)-x_{t+1}^{i}\right)$ is differentiable, it holds if

$\frac{\partial^{2} \beta\left(x_{t}^{i}\right) u\left[g^{i}\left(x_{t}^{i}, x_{t}^{j}\right)-x_{t+1}^{i}\right]}{\partial x_{t}^{i} \partial x_{t+1}^{i}} \geq 0$.

(iii) Let us now prove the increasing differences. A function $f$ has increasing differences if and only if

$f\left(x, t^{\prime \prime}\right)-f\left(x, t^{\prime}\right), \quad t^{\prime \prime}>t^{\prime}$,

is increasing in $x$.

Let $\boldsymbol{x}_{i} \in B r^{i}\left(\boldsymbol{x}_{j}\right)$ so that $x_{t+1}^{i} \in \mu^{i}\left(x_{t}^{i} \mid \boldsymbol{x}_{j}\right), \forall t$. As open-loop strategies are only dependent on the initial conditions, one can easily write that

$$
x_{t}^{i}=\underbrace{\mu^{i}\left(\mu^{i}\left(\mu^{i}\left(\ldots \mu^{i}\left(x_{0}^{i}\right)\right)\right) \mid \boldsymbol{x}_{j}\right)}_{t \text {-times }} \equiv \mu_{t}^{i}\left(x_{0}^{i} \mid \boldsymbol{x}_{j}\right),
$$

$\forall t=1,2, \ldots$
Now we fix $\hat{\boldsymbol{x}}_{j}>\boldsymbol{x}_{j}$ (that is $\hat{x}_{t}^{j}>x_{t}^{j}$ for all $t$ ), and consider a best response of agent $i, \hat{\boldsymbol{x}}_{i} \equiv\left\{\hat{x}_{t}^{i}\right\}_{t=1}^{\infty}$ so that $\hat{x}_{t+1}^{i} \in \hat{\mu}^{i}\left(\hat{x}_{t}^{i} \mid \hat{\boldsymbol{x}}_{j}\right), \forall t$. Accordingly,

$$
\hat{x}_{t}^{i}=\underbrace{\hat{\mu}^{i}\left(\hat{\mu}^{i}\left(\hat{\mu}^{i}\left(\ldots \hat{\mu}^{i}\left(x_{0}^{i}\right)\right)\right) \mid \hat{\boldsymbol{x}}_{j}\right)}_{t \text {-times }} \equiv \hat{\mu}_{t}^{i}\left(x_{0}^{i} \mid \hat{\boldsymbol{x}}_{j}\right),
$$

$\forall t=1,2, \ldots$

We have to check whether

$U\left(\hat{\boldsymbol{x}}_{i}, \hat{\boldsymbol{x}}_{j}\right)-U\left(\hat{\boldsymbol{x}}_{i}, \boldsymbol{x}_{j}\right) \geq U\left(\boldsymbol{x}_{i}, \hat{\boldsymbol{x}}_{j}\right)-U\left(\boldsymbol{x}_{i}, \boldsymbol{x}_{j}\right)$

for $\hat{\mu}^{i}\left(x \mid \hat{\boldsymbol{x}}_{j}\right)>\mu^{i}\left(x \mid \boldsymbol{x}_{j}\right), \forall x$. We can substitute $U$ :

$$
\begin{aligned}
\sum_{t=0}^{\infty} & \left(\prod_{s=1}^{t} \beta\left(\hat{\mu}_{s}^{i}\left(x_{0}^{i}\right)\right)\right)\left[u\left(g^{i}\left(\hat{\mu}_{t}^{i}\left(x_{0}^{i}\right), \hat{x}_{t}^{j}\right)-\hat{\mu}_{t+1}^{i}\left(x_{0}^{i}\right)\right)\right. \\
& \left.-u\left(g^{i}\left(\hat{\mu}_{t}^{i}\left(x_{0}^{i}\right), x_{t}^{j}\right)-\hat{\mu}_{t+1}^{i}\left(x_{0}^{i}\right)\right)\right] \\
\geq & \sum_{t=0}^{\infty}\left(\prod_{s=1}^{t} \beta\left(\mu_{s}^{i}\left(x_{0}^{i}\right)\right)\right)\left[u\left(g^{i}\left(\mu_{t}^{i}\left(x_{0}^{i}\right), \hat{x}_{t}^{j}\right)-\mu_{t+1}^{i}\left(x_{0}^{i}\right)\right)\right. \\
& \left.-u\left(g^{i}\left(\mu_{t}^{i}\left(x_{0}^{i}\right), x_{t}^{j}\right)-\mu_{t+1}^{i}\left(x_{0}^{i}\right)\right)\right] .
\end{aligned}
$$

Since $\hat{\mu}_{s}^{i}\left(x_{0}^{i}\right) \geq \mu_{s}^{i}\left(x_{0}^{i}\right)$ for all $s$, we have that the above inequality is equivalent to checking whether:

$$
\begin{aligned}
& \beta\left(\hat{\mu}_{t}^{i}\left(x_{0}^{i}\right)\right) u\left(g^{i}\left(\hat{\mu}_{t}^{i}\left(x_{0}^{i}\right), \hat{x}_{t}^{j}\right)-\hat{\mu}_{t+1}^{i}\left(x_{0}^{i}\right)\right) \\
& \quad-\beta\left(\hat{\mu}_{t}^{i}\left(x_{0}^{i}\right)\right) u\left(g^{i}\left(\hat{\mu}_{t}^{i}\left(x_{0}^{i}\right), x_{t}^{j}\right)-\hat{\mu}_{t+1}^{i}\left(x_{0}^{i}\right)\right) \\
& \geq \beta\left(\mu_{t}^{i}\left(x_{0}^{i}\right)\right) u\left(g^{i}\left(\mu_{t}^{i}\left(x_{0}^{i}\right), \hat{x}_{t}^{j}\right)-\mu_{t+1}^{i}\left(x_{0}^{i}\right)\right) \\
& \quad-\beta\left(\mu_{t}^{i}\left(x_{0}^{i}\right)\right) u\left(g^{i}\left(\mu_{t}^{i}\left(x_{0}^{i}\right), x_{t}^{j}\right)-\mu_{t+1}^{i}\left(x_{0}^{i}\right)\right) .
\end{aligned}
$$

We shall proceed by dividing both sides by $\left(\hat{x}_{t}^{j}-x_{t}^{j}\right)$ and taking the limit when $\left(\hat{x}_{t}^{j}-x_{t}^{j}\right) \rightarrow 0$. We get that (13) holds if and only if

$$
\begin{aligned}
& \frac{\partial\left[\beta\left(\hat{\mu}_{t}^{i}\left(x_{0}^{i}\right)\right) u\left(g^{i}\left(\hat{\mu}_{t}^{i}\left(x_{0}^{i}\right), \hat{x}_{t}^{j}\right)-\hat{\mu}_{t+1}^{i}\left(x_{0}^{i}\right)\right)\right]}{\partial x_{t}^{j}} \\
& -\frac{\partial\left[\beta\left(\mu_{t}^{i}\left(x_{0}^{i}\right)\right) u\left(g^{i}\left(\mu_{t}^{i}\left(x_{0}^{i}\right), x_{t}^{j}\right)-\mu_{t+1}^{i}\left(x_{0}^{i}\right)\right)\right]}{\partial x_{t}^{j}} \geq 0 .
\end{aligned}
$$

Note that when $\left(\hat{\mu}^{i}\left(x_{0}^{i}\right)-\mu^{i}\left(x_{0}^{i}\right)\right) \rightarrow 0$, then $\left(\hat{\mu}_{s}^{i}\left(x_{0}^{i}\right)-\mu_{s}^{i}\left(x_{0}^{i}\right)\right)$ $\rightarrow 0, \forall s$. Dividing both sides by $\left(\hat{\mu}_{t}^{i}\left(x_{0}^{i}\right)-\mu_{t}^{i}\left(x_{0}^{i}\right)\right)$ and taking the limit when $\left(\hat{\mu}_{t}^{i}\left(x_{0}^{i}\right)-\mu_{t}^{i}\left(x_{0}^{i}\right)\right) \rightarrow 0$, we obtain that

$$
\frac{\partial^{2}\left[\beta\left(\mu_{t}^{i}\left(x_{0}^{i}\right)\right) u\left(g^{i}\left(\mu_{t}^{i}\left(x_{0}^{i}\right), x_{t}^{j}\right)-\mu_{t+1}^{i}\left(x_{0}^{i}\right)\right)\right]}{\partial\left(\mu_{t}^{i}\left(x_{0}^{i}\right)\right) \partial x_{t}^{j}} \geq 0 .
$$

Put differently, in terms of period utilities,

$$
\frac{\partial^{2}\left[\beta\left(x_{t}{ }^{i}\right) u\left(g^{i}\left(x_{t}^{i}, x_{t}^{j}\right)-x_{t+1}^{i}\right)\right]}{\partial x_{t}^{i} \partial x_{t}^{j}} \geq 0,
$$

together with

$\frac{\partial^{2}\left[\beta\left(x_{t}{ }^{i}\right) u\left(g^{i}\left(x_{t}{ }^{i}, x_{t}^{j}\right)-x_{t+1}^{i}\right)\right]}{\partial x_{t}^{i} \partial x_{t+1}^{i}} \geq 0$,

ensures the supermodularity of the game. Since $\mathbf{S}$ is compact under product topology and $U^{i}$ is upper semicontinuous in $\boldsymbol{x}_{i}$ on $S^{i}\left(\boldsymbol{x}_{j}\right)$, the result follows from Theorem 1. 


\section{A.2. Proof of Proposition 9}

By the same reasoning that we use in the proof of Proposition 8(iii), condition (10) assures that for each player, $U^{\tau i}$ has increasing differences in $\left(\boldsymbol{x}_{i}, \tau\right)$. All the rest follows from Topkis (1998, Theorem 4.2.2).

\section{A.3. Proof of Proposition 10}

We proceed in three steps. First, we show that the game $\left(N, \mathbf{S},\left\{U^{i}: i \in N\right\}\right)$ only admits symmetric and antisymmetric steady states. Then, we prove that antisymmetric steady states are linearly ordered with the highest and the lowest symmetric steady states. Finally, we prove that our game only admits symmetric steady state open-loop Nash equilibria.

Lemma 2. If $\left(\boldsymbol{x}_{*}, \boldsymbol{x}^{*}\right)$ is a steady state open-loop Nash equilibrium of the game $\left(N, \mathbf{S},\left\{U^{i}: i \in N\right\}\right)$, then $\left(\boldsymbol{x}^{*}, \boldsymbol{x}_{*}\right)$ is also a steady state open-loop Nash equilibrium.

Proof. If $\left(\boldsymbol{x}_{*}, \boldsymbol{x}^{*}\right)$ is a steady state open-loop Nash equilibrium of the game $\left(N, \mathbf{S},\left\{U^{i}: i \in N\right\}\right)$, then $\left(x_{*}, x^{*}\right)$ satisfies (11) for both agents. $\left(x^{*}, x_{*}\right)$ is also a steady state since for each $i, j \in N, i \neq$ $j, g^{i}\left(x^{i}, x^{j}\right)=g^{j}\left(x^{j}, x^{i}\right)$ and $\frac{\partial g^{i}}{\partial x^{i}}=\frac{\partial g^{j}}{\partial x^{j}}$.

Lemma 2 shows that the set of steady states only contains symmetric and antisymmetric steady states. As the next lemma shows, the antisymmetric steady states are linearly ordered with the highest and the lowest symmetric steady states.

Lemma 3. Let $(\hat{\boldsymbol{x}}, \hat{\boldsymbol{x}})$ and $(\check{\boldsymbol{x}}, \check{\boldsymbol{x}})$ denote the highest and the lowest symmetric steady state open-loop Nash equilibrium of the supermodular game $\left(N, \mathbf{S},\left\{U^{i}: i \in N\right\}\right)$. If there exists an asymmetric steady state open-loop Nash equilibrium, say $\left(\boldsymbol{x}_{*}, \boldsymbol{x}^{*}\right)$ with $\boldsymbol{x}^{*} \neq \boldsymbol{x}_{*}$, then $(\check{\boldsymbol{x}}, \check{\boldsymbol{x}}) \leq\left(\boldsymbol{x}_{*}, \boldsymbol{x}^{*}\right) \leq(\hat{\boldsymbol{x}}, \hat{\boldsymbol{x}})$

Proof. Let $x_{*}<x^{*}$, without loss of generality. Let $x_{0}$ be such that $g^{i}\left(x_{0}, x_{0}\right)=\min \left\{g^{i}\left(x^{*}, x_{*}\right), g^{j}\left(x^{*}, x_{*}\right)\right\}$ for $i \in\{1,2\}$.

Assume now on the contrary that $x_{*}<\tilde{x}^{*}$. We have, by construction,

$g^{i}\left(x_{0}, x_{0}\right)<g^{i}\left(x_{*}, x_{*}\right)<g^{i}\left(\breve{x}^{*}, \breve{x}^{*}\right)$.

Let $\tau=\left(g^{i}\left(x_{0}, x_{0}\right), g^{j}\left(x_{0}, x_{0}\right)\right)$ and $\breve{\tau}=\left(g^{i}\left(x^{*}, x_{*}\right), g^{j}\left(x^{*}, x_{*}\right)\right)$, where $\tau<\breve{\tau}$. Consider the corresponding supermodular games $\Gamma(\tau)$ and $\Gamma(\tilde{\tau})$. By Proposition 9, the greatest and the least equilibrium of the game are increasing in $\tau$ on $T$, i.e. steady state levels that the greatest and the least open-loop Nash equilibrium of the game converge to will be higher for $\breve{\tau}$. Since $\Gamma(\tau)$ is a symmetric supermodular game, the least equilibrium converges to a symmetric steady state, i.e. $\breve{x}^{*} \leq x_{*}$, leading to a contradiction. The case of $x^{*} \leq \hat{x}^{*}$ can be shown similarly.

Let us prove now that any steady state open-loop Nash equilibrium of our game is indeed symmetric:

Let $(\hat{\boldsymbol{x}}, \hat{\boldsymbol{x}})$ and $(\check{\boldsymbol{x}}, \check{\boldsymbol{x}})$ denote the highest and the lowest symmetric steady state open-loop equilibrium of the game, respectively. First and as a corollary to Lemma 3, if the highest and the lowest symmetric steady states coincide, i.e., $\hat{\boldsymbol{x}}=\check{\boldsymbol{x}}$, there does not exist any asymmetric stationary state open-loop equilibrium of the game. Now consider the case where $\hat{\boldsymbol{x}} \neq \check{\boldsymbol{x}}$ and assume that $\left(\boldsymbol{x}_{*}, \boldsymbol{x}^{*}\right)$ is an asymmetric stationary state openloop Nash equilibrium with $\boldsymbol{x}_{*}<\boldsymbol{x}^{*}$ without loss of generality. Then, by Lemma $2,\left(\boldsymbol{x}^{*}, \boldsymbol{x}_{*}\right)$ constitutes an asymmetric steady state open-loop Nash equilibrium as well. Consider now the game $\left(N, \mathbf{S},\left\{U^{i}: i \in N\right\}\right)$ under the following three initial endowments: $\left(\boldsymbol{x}_{*}, \boldsymbol{x}^{*}\right),\left(\boldsymbol{x}^{*}, \boldsymbol{x}_{*}\right)$ and $\left(\frac{x^{*}+x_{*}}{2}, \frac{x^{*}+x_{*}}{2}\right)$. Recall that the game with a symmetric initial endowment turns out to be symmetric supermodular so that due to the monotonicity of the best replies, the greatest and the least equilibrium converge to the highest and the lowest symmetric steady state, respectively. Accordingly, assume that the equilibrium of the symmetric supermodular game that starts with the initial endowment of $\left(\frac{x^{*}+x_{*}}{2}, \frac{x^{*}+x_{*}}{2}\right)$ converges to the highest symmetric steady state $(\hat{\boldsymbol{x}}, \hat{\boldsymbol{x}})$ in the long run, without loss of generality. In comparison with the asymmetric stationary state open-loop equilibrium $\left(\boldsymbol{x}_{*}, \boldsymbol{x}^{*}\right)$, the open-loop Nash equilibrium of the game that emanates from $\left(\frac{x^{*}+x_{*}}{2}, \frac{x^{*}+x_{*}}{2}\right)$ and converging to $(\hat{\boldsymbol{x}}, \hat{\boldsymbol{x}})$ in the long run reveals that an increase in the agent's own initial capital stock and a decrease in the rival's implies the convergence of the agent's stock towards a higher steady state. This then implies that the equilibrium of the supermodular game that emanates from $\left(\boldsymbol{x}^{*}, \boldsymbol{x}_{*}\right)$ has to monotonically converge to the highest steady state $(\hat{\boldsymbol{x}}, \hat{\boldsymbol{x}})$ as well. However, this contradicts the fact that $\left(\boldsymbol{x}^{*}, \boldsymbol{x}_{*}\right)$ has to be a steady state open-loop Nash equilibrium.

\section{A.4. Proof of Proposition 11}

Let $x_{0}$ be such that

$g^{i}\left(x_{0}, x_{0}\right)=\min \left\{f\left(x_{L}\right), \frac{f\left(2 x_{L}^{o}\right)}{2}\right\}, \quad i \in\{1,2\}$,

and consider the corresponding symmetric supermodular game. Let $\left\{x_{0}^{j}, \boldsymbol{x}_{j}\right\}=\left(x_{0}, 0,0 \ldots, 0, \ldots\right)$. Note that $\mathrm{Br}^{i}\left(\boldsymbol{x}_{j}\right)$ coincides with the single agent optimal growth problem for the given initial capital stock, $f^{-1}\left(g^{i}\left(x_{0}, x_{0}\right)\right)$. As $x_{L}$ is stable from the left, $\exists x_{i} \in$ $B r^{i}\left(\boldsymbol{x}_{j}\right)$ such that $x_{\boldsymbol{i}} \rightarrow x_{L}$. Since $x_{L}$ is the lowest steady state and the optimal policy correspondence of a single agent optimal growth problem is ordered (see Erol et al., 2011), we can conclude that $x_{i}$ is the least element of the best response correspondence. Let $\left(x^{*}, x^{*}\right)$ constitute the least open-loop Nash equilibrium of the game, i.e. $x^{*} \in B r^{i}\left(\boldsymbol{x}^{*}\right)$ and $x^{*} \in B r^{j}\left(\boldsymbol{x}^{*}\right)$. Note that the supermodular game structure implies that the least and the greatest elements of the best response correspondence are increasing in the opponent's strategy. Having $x_{j}<x^{*}$ by construction, we conclude that $x^{*} \geq x_{i}$ and $x_{L}^{0} \geq x_{L}$.

For the second case, let $x_{0}$ be such that

$g^{i}\left(x_{0}, x_{0}\right)=\max \left\{\frac{f\left(2 x_{L}^{o}\right)}{2}, f\left(x_{H}\right)\right\}, \quad i \in\{1,2\}$,

and consider the corresponding symmetric supermodular game. Let $\left\{x_{0}^{j}, \boldsymbol{x}_{j}\right\}=\left(x_{0}, 0,0 \ldots, 0, \ldots\right) . \mathrm{Br}^{i}\left(\boldsymbol{x}_{j}\right)$ coincides with the single agent optimal growth problem for given initial capital stock, $f^{-1}\left(g^{i}\left(x_{0}, x_{0}\right)\right)$. As $x_{H}$ is stable from the right, $\exists x_{\boldsymbol{i}} \in \mathrm{Br}^{i}\left(\boldsymbol{x}_{j}\right)$ such that $x_{i} \rightarrow x_{H}$. By the same reasoning as above, $x_{i}$ is the highest element of the $\mathrm{Br}^{i}\left(\boldsymbol{x}_{j}\right)$. Having $x_{j}<x^{*}$ by construction, we get $x^{*} \geq x_{i}$ and $x_{L}^{0} \geq x_{H}$.

\section{A.5. Proof of Corollary 1}

As proven in Proposition 10, there only exist symmetric steady state open-loop Nash equilibria. At any steady state open-loop Nash equilibrium $(\boldsymbol{x}, \boldsymbol{x})$, agents' payoff can be recast as

$U(\boldsymbol{x} \mid \boldsymbol{x})=u(g(x, x)-x) \frac{1}{1-\beta(x)}$.

Note that if

$\frac{\partial}{\partial x}\left(\frac{u(g(x, x)-x)}{1-\beta(x)}\right)>0$,

then $U(\hat{\boldsymbol{x}} \mid \hat{\boldsymbol{x}}) \geq U(\boldsymbol{x} \mid \boldsymbol{x}) \geq U(\check{\boldsymbol{x}} \mid \check{\boldsymbol{x}})$ holds where at least one of the inequalities will be strict. 


\section{References}

Amir, R., 2005. Supermodularity and complementarity in economics: an elementary survey. Southern Economic Journal 71 (3), 636-660.

Amir, R., Jakubczyk, M., Knauff, M., 2008. Symmetric versus asymmetric equilibria in symmetric supermodular games. International Journal of Game Theory 37 (3), 307-320.

Amir, R., Mirman, L.J., Perkins, W.R., 1991. One-sector nonclassical optimal growth: optimality conditions and comparative dynamics. International Economic Review 32 (3), 625-644.

Araujo, A., 1991. The once but not twice differentiability of the policy function. Econometrica 59 (5), 1383-1393.

Azariadis, C., Stachurski, J., 2005. Poverty traps. In: Aghion, P., Durlauf, S. (Eds.), Handbook of Economic Growth. Elsevier, Amsterdam.

Barro, R.J., 1997. Determinants of Economic Growth. MIT Press, Cambridge, MA.

Barro, R.J., Sala-i-Martin, X., 1991. Convergence across states and regions. Brookings Papers on Economic Activity 1, 107-182.

Becker, G.S., Mulligan, C.B., 1997. The endogenous determination of time preference. The Quarterly Journal of Economics 112 (3), 729-758.

Benveniste, L.M., Scheinkman, J.A., 1979. On the differentiability of the value function in dynamic models of economies. Econometrica 47, 727-732.

Cooper, R., 1999. Coordination Games: Complementarities and Macroeconomics. Cambridge University Press, Cambridge.

Debreu, G., 1952. A social equilibrium existence theorem. Proceedings of the National Academy of Sciences 38, 886-893.

Dechert, W.D., Nishimura, K., 1983. A complete characterization of optimal growth paths in an aggregated model with non-concave production function. Journal of Economic Theory 31, 332-354.
Dockner, E.J., Nishimura, K., 2001. Characterization of equilibrium strategies in a class of difference games. Journal of Difference Equations and Applications 7, 915-926.

Erol, S., Le Van, C., Saglam, C., 2011. Existence, optimality and dynamics of equilibria with endogenous time preference. Journal of Mathematical Economics 47 (2), 170-179.

Lawrence, E.C., 1991. Poverty and the rate of time preference: evidence from panel data. Journal of Political Economy 99, 54-75.

Le Van, C., Dana, R.A., 2003. Dynamic Programming in Economics. Kluwer Academic Publishers.

Mitra, T., Ray, D., 1984. Dynamic optimization on non-convex feasible set: some general results for non-smooth technologies. Zeitschrift für Nationalökonomie 44, 151-175.

Quah, D.T., 1996. Convergence empirics across economies with (some) capital mobility. Journal of Economic Growth 1, 95-124.

Samwick, A., 1998. Discount rate homogeneity and social security reform. Journal of Development Economics 57, 117-146.

Schumacher, I., 2011. Endogenous discounting and the domain of the felicity function. Economic Modelling 28, 574-581.

Stern, M.L., 2006. Endogenous time preference and optimal growth. Economic Theory 29, 49-70.

Stokey, N.L., Lucas, R., Prescott, E., 1989. Recursive Methods in Economic Dynamics. Harvard University Press.

Sundaram, R.K., 1989. Perfect equilibrium in non-randomized strategies in a class of symmetric dynamic games. Journal of Economic Theory 47, 153-177.

Topkis, D.M., 1998. Supermodularity and Complementarity. Princeton University Press.

Vives, X., 2005. Games with strategic complementarities: new applications to industrial organization. International Journal of Industrial Organization 23, 625-637. 